\title{
Transmutation of the vicinal surface exponent due to gravity
}

\author{
J. E. Avron \\ California Institute of Technology (114-36), Pasadena, California 91125 \\ and Department of Physics, Technion-Israel Institute of Technology, 32000 Haifa, Israel \\ R. K. P. Zia \\ Center for Transport Theory and Mathematical Physics, Department of Physics, \\ Virginia Polytechnic Institute and State University, Blacksburg, Virginia 24061-4097
}

(Received 9 October 1987)

\begin{abstract}
Near the edge of a facet, vicinal surfaces curve away from the plane with exponent $\frac{3}{2}$. With gravity normal to the facet the exponent may change to 3 . Generally, there will be a crossover from 3 to $\frac{3}{2}$ as the facet edge is approached. Comparison with experiments is made.
\end{abstract}

Vicinal surfaces are those parts of a crystalline interface that join smoothly to a facet. The limiting behavior is described by the vicinal exponent $\theta$, defined by

$$
\delta y \sim(\delta x)^{\theta}
$$

where $\delta y$ is the distance of the surface from the facet plane and $\delta x$ is the distance from the facet edge. See Fig. 1. One reason why this number is interesting is that interface theories ${ }^{1}$ make definite predictions about $\theta$ and measurements can be used to single out the more successful ones. Classical, mean-field theory gives $\theta=2$ (at thermal equilibrium). ${ }^{2}$ Solid-on-solid (SOS) lattice-type models give $\theta=\frac{3}{2} .^{3}$

Several experiments on small metallic crystals support $\theta=\frac{3}{2} .{ }^{4}$ Recent, precise measurements of Carmi et al. ${ }^{5}$ for macroscopic He crystals, where the facet is oriented vertically, give $\theta=1.55 \pm 0.06$ and exclude $\theta=2$ by a large margin. On the other hand, older measurements of Babkin et al. ${ }^{6}$, also for macroscopic He crystals, but with the facet oriented horizontally, are inconsistent with $\theta=\frac{3}{2}$. Although the results are not in good agreement with $\theta=2$ either, $\theta=2$ fits the data better than $\theta=\frac{3}{2}$. As pointed out by Carmi, Lipson, and Polturak, ${ }^{5}$ the two $\mathrm{He}$ results need not be inconsistent. In a gravity-free envi-

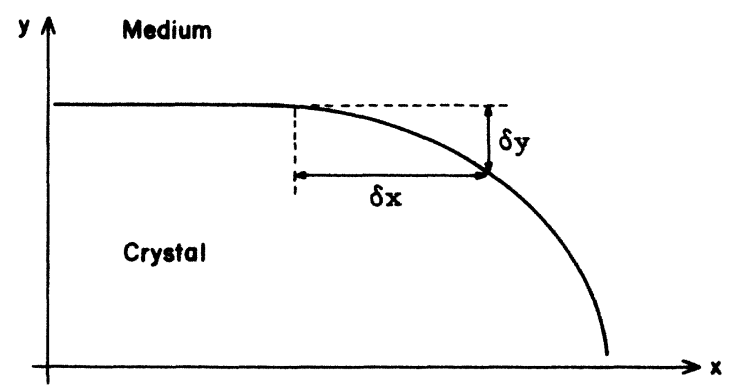

FIG. 1. Schematic drawing of a crystal near a facet edge. $\delta y$ and $\delta x$ are the vertical and horizontal distances from the facet edge. Gravity is assumed to point along the $y$ axis. ronment, or for crystals small compared with the capillary length, horizontal and vertical facets have the same vicinal exponent. However, for large crystals in a gravitational field this need not be the case. They suggested that there are no gravitational corrections to the vicinal exponents of vertical facets, and there are such corrections for horizontal facets that tend to increase $\theta$. One of our purposes is to show that this is indeed the case and make predictions regarding the values $\theta$ takes. As we shall show, horizontal facets have a crossover from $\theta$ close to the edge to $\theta /(2-\theta)$ away from it, provided $\theta<2$. For $\theta=2$, the crossover it to an exponential. The crossover region is given by $\delta y \sim \Delta(V, g)$, a length depending on the volume $V$ and the gravitational acceleration $g$. If $\theta<2$, at least in two dimensions, $\Delta$ will vanish identically if the crystal is big enough. In this case the exponent $\theta$ is transmuted to $\theta /(2-\theta)$ all the way to the edge. In particular, $\theta=\frac{3}{2}$ is transmuted to 3 . In three dimensions $\Delta$ is a decreasing function of the volume. We do not know if or when it can actually vanish identically for large enough crystals. This is related to the fact that equilibrium shapes with gravity do not admit analytic solutions in three dimensions.

In this light, we have reanalyzed the data of Babkin et $a l .{ }^{6}$ and found good agreement with $\theta=3$. We conclude that the experiments of Carmi et al. and Babkin et al. agree and both support the SOS result of $\theta=\frac{3}{2}$ for a gravity-free interface and disagree with the mean-field prediction of $\theta=2$.

Let us introduce notation and state the basic assumptions of the theoretical framework. Denote by $y(x)$ the crystal shape and $\mathrm{p}=\boldsymbol{\nabla} \boldsymbol{y}$ the tangent. The surface free energy is $\sigma(p)$ and we define $f(p) \equiv\left(1+p^{2}\right)^{1 / 2} \sigma(p)$. In two dimensions the crystal is a film or a slab and the interface a line. $x$ and $p$ are then scalars. In three dimensions the interface is a surface and $x$ and $p$ are vectors in the plane. Let $|p|$ be the length of $p$ and suppose that $\mathbf{p}=\mathbf{0}$ is the direction of the facet. Also $\mathcal{L}_{f}$ denotes the Ledendre transform of $f$, i.e., $\mathcal{L}_{f}(\mathbf{p}) \equiv \mathbf{p} \cdot \nabla f-f$.

Interface theories make predictions about the $\mathbf{p}$ dependence of the surface tension near a facet. Expressed in 
terms of the Legendre transform of $f$, the issue is what is $\mu$ in the expansion of $\mathcal{L}_{f}(\mathbf{p})$ for $|\mathbf{p}|$ small:

$$
\mathcal{L}_{f}(\mathbf{p})=-f(0)+C(\hat{\mathbf{p}})|\mathbf{p}|^{\mu}+\cdots,
$$

where $\hat{\mathbf{p}}=\mathbf{p} /|\mathbf{p}|$. If $f(\mathbf{p})$ has an expansion in $|\mathbf{p}|$ then $\mu$ must be an integer, and it must be at least 2 since the Legendre transform eliminates the linear piece. Meanfield theory asserts the naive expectation $\mu=2$. SOS models, on the other hand, predict a nontrivial cancellation of the quadratic piece give $\mu=3$.

In the absence of gravity, $\mu$ and the vicinal exponent $\theta$ are related by the Wulff construction. ${ }^{7}$ The equilibrium shape is $y=\mathcal{L}_{f}(\mathbf{p})$, from which it follows that

$$
\theta=\mu /(\mu-1) \text {. }
$$

$A$ priori, gravity could modify the analysis in two ways: First, it could change the asymptotic behavior of $\mathcal{L}_{f}(\mathbf{p})$. [For example, if the coefficient of the quadratic term in Eq. (1) is proportional to $g, \mu=3$ if $g=0$ and $\mu=2$ if $g \neq 0$.] Second, the relation between $\mu$ and $\theta$, Eq. (2), needs to be modified since the equilibrium shape is no longer given by the Wulff construction.

The basic assumption we shall make is that $\sigma(p)$ is independent of $g$, which implies that $\mu$ is independent of $g$. This is related to the continuum limit. Explicitly, on height scales much smaller than the capillary length, gravity effects are small. If this scale is still much larger than interatomic distances, $\sigma(p)$ may be defined locally, according to the usual statistical mechanics definition. Thus, in the continuum limit where interatomic distances are negligible, $\sigma(\mathbf{p})$ is a local property, independent of $g$. In the rest of this paper we examine how Eq. (2) is modified by gravity.

It is actually somewhat surprising that gravity can transmute the vicinal exponent at all. For the vicinal exponent [like $\sigma(\mathbf{p})$ ] appears to be a local property that is defined on height scales that can be much smaller than capillary lengths. This naive picture is false. The vicinal exponent actually depends on global features like the volume and actual height of the crystal. To see this, recall the Euler-Lagrange equation for the minimization of the surface-free energy, and the gravitational energy subject to a volume constraint (Herring's formula ${ }^{8,9}$ ):

$$
-\sum \frac{\partial^{2} y}{\partial x_{i} \partial x_{j}} \frac{\partial^{2} f}{\partial p_{i} \partial p_{j}}=\kappa(\Delta+\delta y),
$$

where $\kappa \equiv(\Delta \rho) g$ is the "specific weight" of the crystal ( $\Delta \rho$ is the difference in densities between the crystal and the surrounding medium), $\Delta \equiv(\lambda / \kappa)-h$ where $h$ is the height of the crystal, $\delta y \equiv h-y$, and $\lambda>0$ is the Lagrange multiplier associated with the volume constraint. The "source term," the right-hand side of (3), is non-negative, expressing the convexity of the equilibrium shape. If $g=0$, or if $\Delta>0$ and $\delta y \ll \Delta$, the "source" is essentially a fixed strictly positive constant, so the vicinal exponents are the same. However, when $\Delta$ vanishes (or when $\delta y$ dominates $\Delta$ ), the source is qualitatively different and becomes arbitrarily weak as $\delta y$ gets small. There is no reason why the vicinal exponent in such a case would be as in the $g=0$ case. Since $\Delta$ depends on global features ( $h$ and $V$ ) $\theta$ is not a purely local property.

The vicinal exponent of vertical surfaces is not modified by gravity because for such surfaces $\delta y$ does not get to be arbitrarily small. But that of horizontal surfaces may be modified. Before discussing this modification quantitatively, let us give an interpretation of the crossover scale $\Delta$.

The gravity-free equilibrium crystal surface can be understood as the balance between the pressure difference across the surface and the (anisotropic) surface energy. ${ }^{10}$ It is intuitively obvious that the crystal shape is convex if the pressure is higher on the crystal side. In the presence of gravity, the effective pressure is linear in $y$ and vanishes at some "fiducial" height. The crystal surface clearly does not rise above this height; otherwise it would be concave. $\Delta$ is precisely the difference between the fiducial height and the maximum height of the crystal. For crystal surfaces which are rounded at the top, Herring's formula ${ }^{8,9}$ states $\kappa \Delta=\sum K_{i} S_{i}$, where $K_{i}$ are the principal curvatures at the top and $S_{i}$ are principal radii of curvature in the Wulff plot there (i.e., the eigenvalues of the stiffness tensor ${ }^{11}$ ). Since $S_{i}$ remain fixed while the curvature of the crystal is expected to decrease for increasing $g$ and $V, \Delta$ is expected to be a decreasing function of $V$. (It may not be monotonically so for the height is known to be nonmonotonic for liquids. $)^{12}$ Thus, $\Delta$ can be made small by enlarging the crystal. For crystals with a facet at the top, this conclusion is not altered, though the arguments need to be tightened, as Herring's formula holds for the smooth portions of the interface.

To examine how $\theta$ changes, consider first the twodimensional case of a crystal supported on a flat horizontal base. The Euler-Lagrange equation has a first integral $^{9}$

$$
\mathcal{L}_{f}(p)+\lambda y-\frac{1}{2} \kappa y^{2}=\mathcal{L}_{f}\left(p_{0}\right),
$$

where $p_{0}$ is the tangent of the contact angle. With a facet at $p=0$, subtracting $y(p)$ from $h=y(0)$ in Eq. (4) one gets

$$
\delta y(2 \Delta+\delta y)=\frac{2}{\kappa}\left[\mathcal{L}_{f}(p)-\mathcal{L}_{f}(0)\right] .
$$

By (1), the right-hand side of (5) is of order $|p|^{\mu}$. Thus, $\delta y \sim p^{\mu}$ if $\Delta \gg \delta$ and $\delta y \sim p^{\mu / 2}$ if $\delta y \gg \Delta$, so that

$$
\theta=\left\{\begin{array}{c}
\mu /(\mu-1) \text { if } \delta y \ll|\Delta| \\
\mu /(\mu-2) \text { if } \delta y \gg|\Delta|
\end{array}\right.
$$

So, there is a crossover at a scale $\Delta$. If $\Delta=0$, the exponent is transmuted all the way to the edge of the facet. In particular, if $\mu=3, \theta=3$ for $\delta y$ larger than $\Delta$ and $\frac{3}{2}$ for $\delta y$ smaller than $\Delta$. From Ref. $9 \lambda$ is a nonincreasing and $h$ a nondecreasing function of $V$ so $\Delta$ is a nondecreasing function of $V$. In fact, if $\mu>2, \Delta$ vanishes for $V>V_{0}$, where $V_{0}$ is the finite critical volume whose part lying to the right of the facet, $V_{0}^{\prime}$, is

$2 \kappa V_{0}^{\prime}=\int_{p_{0}}^{0}\left[\left[1-\frac{2 \kappa}{\lambda^{2}}\left[\mathcal{L}_{f}(p)-\mathcal{L}_{f}\left(p_{0}\right)\right]\right]^{-1 / 2}-1\right] f^{\prime \prime}(p) d p$. 
The three-dimensional case is, of course, more complicated. We restrict ourselves to the case of cylindrical symmetry where the Euler-Lagrange equation is the (nonlinear) differential equation

$$
\frac{1}{\kappa r} \frac{\partial}{\partial r}\left[r f^{\prime}(p)\right]=(\Delta+\delta y) \text {. }
$$

Here $r \equiv|x|, p \equiv \partial y / \partial r$. Let $r_{0}$ be the edge of the facet, $f^{\prime}(p)=s+$ const $\times p^{(\mu-1)}$ and $\delta y \sim(\delta r)^{\theta}$, with $\theta>1(s>0$ is the step-free energy). The left-hand side of $(8)$ is, to leading orders,

$$
\frac{1}{\kappa r_{0}}\left[s+(\text { const })^{\prime}(\delta r)^{(\mu-1)(\theta-1)-1}+\cdots\right] \text {. }
$$

There are two possibilities: Either $r_{0} \Delta=s / \kappa$, in which case $(\mu-1)(\theta-1)-1=\theta$, or $r_{0} \Delta \neq s / \kappa$, in which case $(\mu-1)(\theta-1)-1=0$. The first gives $\theta=\mu /(\mu-2)$ and the second gives $\theta=\mu /(\mu-1)$. Thus, again, depending on $\Delta$ there is a transmutation of $\theta$. Also, as in two dimensions in the general case, there is a crossover.

In light of the transmutation of $\frac{3}{2}$ to 3 , we took the data of Babkin et al., ${ }^{6}$ presented in their Fig. 3b, and plotted $\sqrt{\phi}$ against $x$. Since $\phi$ is essentially $p$ at these angles, $\delta y \sim(\delta x)^{3}$ predicts that our plot should be linear, with a crossover to $(\delta x)^{1 / 4}$. In Fig. 2, we see that the first two points are still on the facet while all but the last two or three lie on a straight line. We cannot quote how well a straight line fits the data, since we have neither the numerical values nor the errors. (One difficulty is that it is not obvious how many points actually lie in the asymptotic region of vicinal surfaces. As pointed out to us by Lipson, using a criterion of Jyaprakash and Saam in Ref. 3 to define the vicinal region, his analysis of the data of Babkin et al. suggests that the scale is about $2 \mathrm{~mm}$, in reasonable agreement with the $2.8 \mathrm{~mm}$ suggested by our fit.) The overall agreement supports the claim of transmutation.

From the graph we can also get an estimate of $\Delta$. The crossover appears to occur between the second point, which is on the facet, and the third point, which is already on the straight line. This corresponds to $\sqrt{\phi}$ of the order of 0.3 and so to $\phi$ of order 0.01 , and $\delta x$ of order of a fraction of a $\mathrm{mm}$. This gives $\delta y$, and $\Delta$ of the order of a micrometer. With more data between the second and the third point, $\Delta$ could be determined more accurately.

It is probably no coincidence that data in the crossover region are not reported. The authors of Ref. 6 pointed

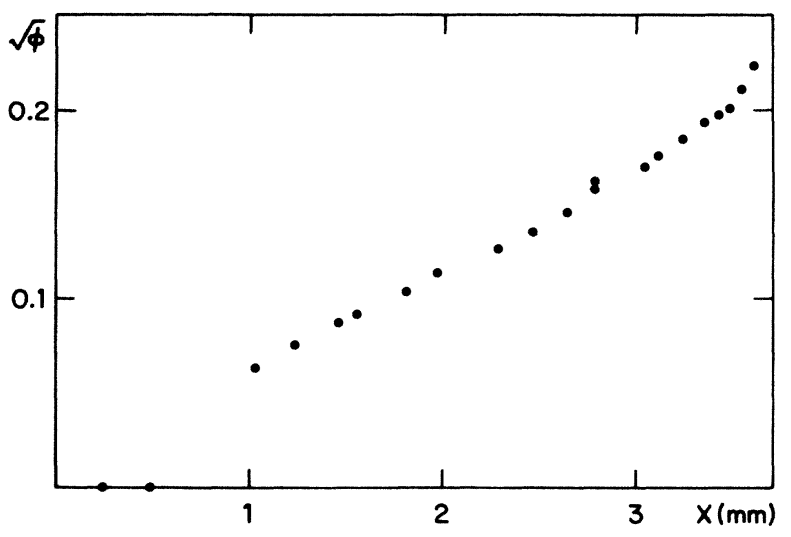

FIG. 2. Replotting of the data of Babkin et al. in Ref. 6, using $\sqrt{\phi}$ instead of their $\phi$. The graph of $\sqrt{\phi}$ as function of $\delta x$ would be a straight line if the gravity-free vicinal exponent $\frac{3}{2}$ undergoes transmutation to 3 .

out the uncertainties concerning the lack of equilibrium ${ }^{13}$ for facets and their neighborhoods. Thus, even if measurements were taken in this region, it would be difficult to untangle the effects of nonequilibrium from the critical behavior of vicinal surfaces. In this sense, there is much theoretical work to be done on the difficult issue of dealing with surfaces in equilibrium joined to those not in equilibrium.

If the external force is not uniform but depends on $y$, it is possible for the exponent to be transmuted some other way. This can be traced to the third term in Eq. (4). If its power were $m$ instead of 2 , which came from the specific form of the gravitational potential, then the transmuted exponent will be $\theta /[m-(m-1) \theta]$. Nonuniform forces can be produced in centrifuges or between uneven condenser plates, for examples.

Finally, similar ideas can be used to analyze the effects of gravity on the universal jump of the curvature near the roughening transition. ${ }^{13,14}$

We thank S. G. Lipson for bringing the problem to our attention and for illuminating discussions. This research is supported in part by grants from the Israel Academy of Sciences and Humanities, the fund for the promotion of research at the Technion, and the U.S. National Science Foundation under Grants No. DMR-85-04716 and No. DMS-84-16049.
${ }^{1}$ D. P. Woodruff, The Solid-Liquid Interface (Cambridge University Press, London, 1973); C. Rottman and M. Wortis, Phys. Rep. 103, 59 (1984); R.K.P. Zia, in Statistical and Particle Physics-Common Problems and Techniques, edited by $\mathbf{K}$. C. Bowler and A. J. McKane (Scottish Universities Summer School in Physics Publications, Edinburgh, 1984), pp. 247-301.

${ }^{2}$ A. F. Andreev, Zh. Eksp. Teor. Fiz. 80, 2042 (1981) [Sov. Phys._JETP 53, 1063 (1982)].
${ }^{3}$ C. Jayaprakash and W. F. Saam, Phys. Rev. B 30, 3916 (1984); C. Jayaprakash, C. Rottman, and W. F. Saam, ibid. 30, 6549 (1984); V. I. Marchenko and A. Ya. Parshin, Zh. Eksp. Teor. Fiz. 31, 767 (1980) [Sov. Phys. -JETP Lett. 31, 724 (1980)]; E. E. Gruber and W. W. Mullins, J. Phys. Chem. Solids 28, 875 (1967); V. L. Pokrovsky and A. L. Talapov, Phys. Rev. Lett. 42, 65 (1979); A. Saxena, T. Ala-Nissilä, and J. D. Gunton, Surf. Sci. 171, 170 (1986). For a recent review, see H. van Beijeren and I. Nolden (unpublished). 
${ }^{4}$ C. Rottman, M. Wortis, J. C. Heyraud, and J.-J. Métois, Phys. Rev. Lett. 52, 1009 (1984); J. C. Heyraud and J.-J. Métois, Surf. Sci. (unpublished); F. Gallet, Ph.D. thesis, Ecole Normale Supérieure, Paris, 1986.

${ }^{5}$ Y. Carmi, S. G. Lipson, and E. Polturak, Phys. Rev. B 36, 1894 (1987).

${ }^{6}$ A. V. Babkin, D. B. Kopeliovitch, and A. Ya. Parishin, Zh. Eksp. Teor. Fiz. 89, 2288 (1985) [Sov. Phys._JETP 62, 1322 (1985)].

${ }^{7}$ G. Wulff, Z. Krist. Mineral. 34, 449 (1901); see also C. Herring, in Structure and Properties of Solid Surfaces, edited by R. Gomer and C. S. Smith (University of Chicago, Chicago, 1953); W. L. Winterbottom, Acta. Metall., 15, 303 (1967).

${ }^{8}$ C. Herring, Phys. Rev. 82, 87 (1951).

${ }^{9}$ J. E. Avron, J. E. Taylor, and R. K. P. Zia, J. Stat. Phys. 33,
493 (1983).

${ }^{10}$ L. D. Landau and E. M. Lifshitz, Statistical Physics (Pergamon, Oxford, 1959).

${ }^{11}$ D. W. Hoffman and J. W. Cahn, Surf. Sci. 31, 369 (1972); J. W. Cahn and D. W. Hoffman, Acta Metall. 22, 1205 (1974); R. K. P. Zia and J. E. Avron, Phys. Rev. B 25, 2042 (1982).

${ }^{12}$ R. Finn, Pacific J. Math. 88, 549 (1980).

${ }^{13}$ P. E. Wolf, F. Gallet, S. Balibar, E. Rolley, and Nozières, J. Phys. (Paris) 46, 1987 (1985); F. Gallet, S. Balibar, and E. Rolley, J. Phys. (Paris) 48, 369 (1987).

${ }^{14}$ C. Jayaprakash, W. F. Saam, and S. Teitel, Phys. Rev. Lett. 50, 2017 (1983); D. S. Fisher and J. D. Weeks, ibid. 50, 1077 (1983); W. J. Blote and H. J. Hilhorst, J. Phys. A 15, L631 (1982); C. Rottman and M. Wortis, Phys. Rev. B 29, 328 (1984). 